\title{
Introduction to the Cancer Center Cessation Initiative Working Groups: Improving Oncology Care and Outcomes by Including Tobacco Treatment
}

\author{
The Cancer Center Cessation Initiative Coordinating Center and Expert Advisory Panel*
}

Cigarette smoking remains the leading cause of preventable cancer and cancer-related deaths in the United States, responsible for roughly $30 \%$ of all cancer deaths, representing more than 150,000 Americans killed by tobacco-caused cancer each year. ${ }^{1,2}$ Among the estimated 1.9 million people who will be diagnosed with cancer in the United States in $2021,{ }^{3}$ many will receive their oncology care at one of the 64 clinical NCI-designated Cancer Centers ${ }^{4}$; approximately $24 \%$ will be cigarette smokers at the time of diagnosis. ${ }^{5}$

Although compelling data have shown that quitting smoking after a cancer diagnosis leads to better health and quality of life outcomes, a 2009 survey reported that only 38\% of NCI Cancer Centers recorded smoking as a vital sign, and fewer than half had dedicated personnel to provide smoking cessation treatment. ${ }^{6}$ Only about half of patients with cancer who smoke and just $15 \%$ of those who have completed treatment are counseled to quit. ${ }^{7}$ These missed opportunities were highlighted in the 2014 Surgeon General's Report, which documented that continuing to smoke after a cancer diagnosis was associated with significant increases in all-cause mortality, cancerspecific mortality, and second primary cancers. ${ }^{2}$ In 2015, NCCN established Clinical Practice Guidelines in Oncology (NCCN Guidelines) for Smoking Cessation, highlighting best practices for assessment and treatment of tobacco dependence in cancer care. ${ }^{8}$ A recent commentary called for tobacco cessation to be designated as the "Fourth Pillar of Cancer Care," in recognition of its vast potential for improving the lives of patients with cancer. ${ }^{9}$ Integrating tobacco cessation into routine cancer care may be particularly beneficial for some racial, ethnic, and sexual and gender minorities; rural residents; those of low socioeconomic status; and underinsured or uninsured populations, because they experience a higher

\footnotetext{
*A complete list of the Cancer Center Cessation Initiative Coordinating Center and Expert Advisory Panel members appears at the end of this article.
}

burden of tobacco-related cancers and chronic diseases. $^{2,10}$ In fact, medically underserved populations are less likely to even be offered tobacco cessation services and less likely to participate even though they report high rates of interest in quitting. ${ }^{11}$

\section{The Cancer Center Cessation Initiative: An NCl Cancer Moonshot Project}

The NCI Cancer Moonshot-funded Cancer Center Cessation Initiative (C3I) was launched in 2017 in response to these challenges. ${ }^{12}$ The short-term goal of C3I is to enhance the capacity of NCI-designated Cancer Centers to promote smoking cessation among patients with cancer. The long-term goal is to implement sustainable smoking cessation treatment programs within Cancer Centers. The ultimate goal is to disseminate lessons learned by the C3I Cancer Centers to the entire cancer care community, from academic medical centers to community-based clinics, helping to ensure that every patient with cancer who smokes receives cessation treatment.

Fifty-two of the 64 clinical and comprehensive NCIdesignated Cancer Centers were selected to participate in C3I (Figure 1). Funded initially as 2-year supplements to each site's Cancer Center Support Grant, C3I required each Cancer Center to take a population-based approach to ensure that every patient with cancer is screened for tobacco use and that every individual who smokes receives evidence-based cessation treatment. This focus on reaching every patient who enters a cancer center provides an opportunity to engage patients from medically underserved populations who may have previously been excluded. ${ }^{11}$ Each Cancer Center developed its own approach to implementation, tailored to its local context and unique population. Programs include varying combinations of intervention components, including in-person, phone, text, and video-based telehealth counseling; Tobacco Quitline/SmokefreeTXT referrals; pharmacotherapy; and Interactive Voice Response (IVR) programs. Some programs engage front-line clinical staff (eg, rooming staff, 


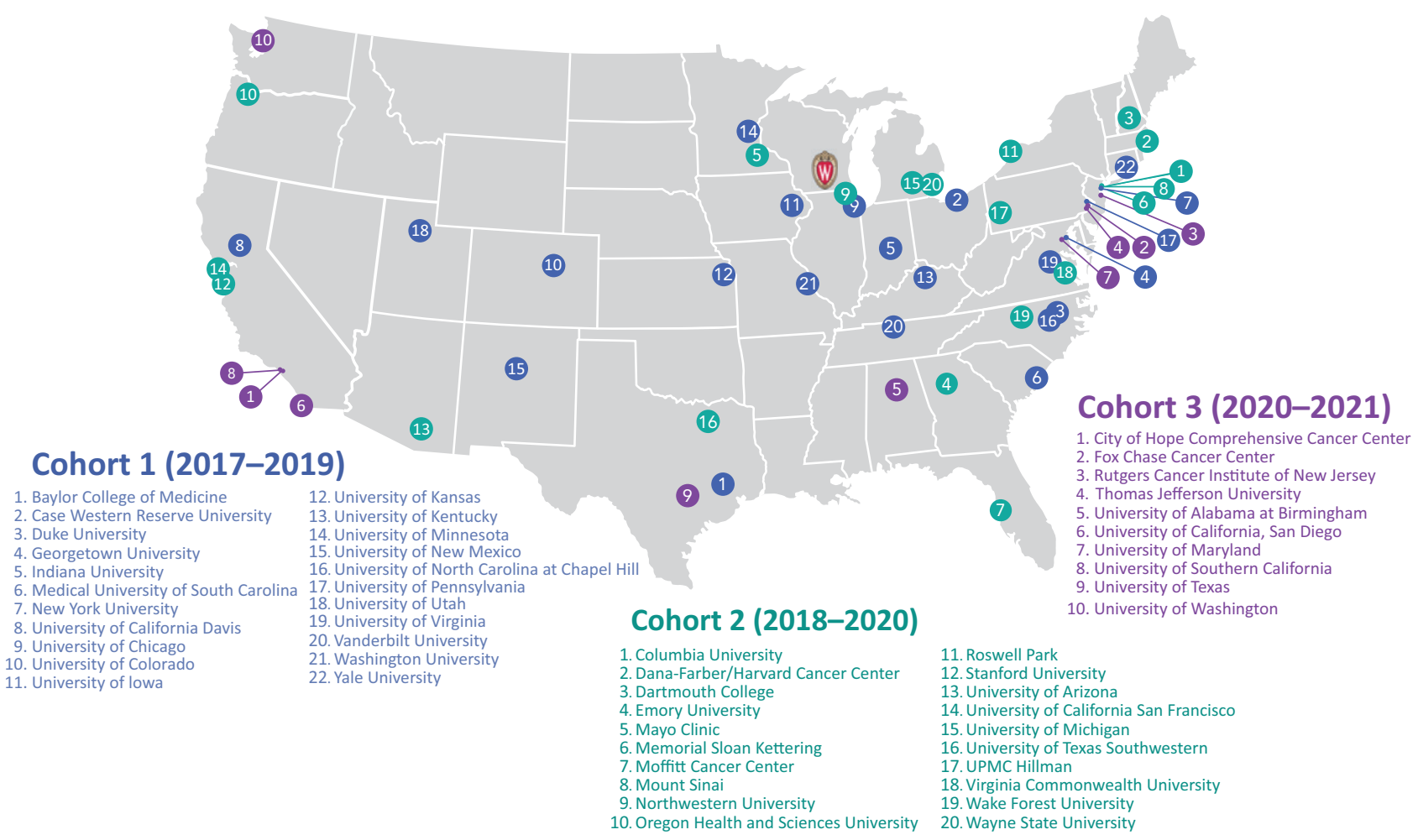

\begin{tabular}{|l|l|l|}
\hline Cohort 1 & $\begin{array}{l}\text { Cohort 2 } \\
\bullet 2018-2020 \\
\bullet 2017-2019 \\
\bullet 22 \text { sites }\end{array}$ \\
$\bullet$ - $250 \mathrm{~K} /$ year for 2 years & $\begin{array}{l}\text { Cohort 3 } \\
\bullet 2020-2021 \\
\bullet 10 \text { sites } \\
\bullet \$ 400 \mathrm{~K} / \text { year for 1 year }\end{array}$ & $\begin{array}{l}\text { Enhancement Funding } \\
\bullet 2020-2021 \\
\bullet 11 \text { Cohort } 1 \text { and } 2 \text { sites } \\
\bullet \$ 200 \mathrm{~K} / \text { year for } 1 \text { year }\end{array}$ \\
\hline
\end{tabular}

Figure 1. C3I-funded cancer centers and timeline.

Abbreviation: $\mathrm{C} 3 \mathrm{l}, \mathrm{NCl}$ Cancer Moonshot-funded Cancer Center Cessation Initiative.

nurses, treating clinicians) to provide cessation treatments, while others rely on tobacco treatment specialists supported by C3I and embedded within the health system. The variety of approaches and settings within C3I allows for an ongoing examination of which types of services and implementation strategies have the greatest reach and effectiveness overall and for specific subgroups of patients.

The goal of the C3I Coordinating Center, housed at the University of Wisconsin Carbone Cancer Center, is to support the integration of evidence-based smoking cessation treatment services into the routine care of the 52 funded centers by: (1) providing technical assistance in the areas of electronic health record (EHR) integration, clinical workflow change management, and addressing health equity; (2) collecting data on reach and effectiveness to monitor the impact of the initiative; and (3) serving as the hub of knowledge integration and dissemination. The Expert Advisory Panel, consisting of clinician-scientists with expertise in tobacco treatment in cancer care, supports the work of the C3I Coordinating Center. The C3I Coordinating Center collects data every 6 months on reach (the percentage of patients with cancer who smoke who engaged in evidence-based cessation treatment) and effectiveness (30day point prevalence abstinence at 6 months among smokers who engaged in treatment) and conducts site visits to collect in-depth qualitative data on implementation strategies and outcomes.

Longitudinal data collected from the 52 C3I sites will be analyzed to identify a broad array of implementation strategies and program characteristics, as well as teamand institutional-level characteristics that impact implementation. ${ }^{13,14}$ Each Center operates in a unique context with all of the complexities of delivering cancer care, making them excellent models for understanding which factors increase or reduce inequities in smoking cessation treatment delivery. C3I has the potential to improve 
oncology care and outcomes by emphasizing the importance of tobacco cessation as an expected component of routine cancer treatment. Over the past 4 years, C3I sites have collectively delivered cessation treatment to more than 50,000 patients with cancer who smoke, more than doubling overall reach from $10.6 \%$ pre-C3I to $22.3 \%$ after C3I funding. Additionally, reach increased from $7 \%$ to $25 \%$ among American Indian/Alaska Natives; from $7 \%$ to $19 \%$ among Asian Americans, Native Hawaiians, and Pacific Islanders; from $19 \%$ to $23 \%$ among Hispanics; and from $19 \%$ to $26 \%$ among Black or African Americans. ${ }^{15}$

\section{C3I Working Groups}

The C3I Coordinating Center, recognizing the potential of the network that emerged from this initiative, solicited research questions from sites in the areas of cessation research, policy, and implementation science that could be uniquely addressed by a sustained C3I Consortium. These questions were categorized into 5 areas, around which the C3I Working Groups were formed in 2020: (1) diversity, equity, and inclusion; (2) family and social support systems; (3) implementation science; (4) sustainability; and (5) telehealth. The Coordinating Center nominated C3I site leaders to serve as Working Group cochairs, paying special attention to demographic and disciplinary diversity to ensure a range of viewpoints and voices are represented. Although each group operates independently, we have identified multiple points of synergy, such as the impact of the COVID-driven move to telehealth on diversity, equity, and inclusion. Since the launch of the Working Groups, each group has met monthly to discuss the relevance of their focus on smoking cessation for patients with cancer who smoke, how C3I sites are addressing each area of focus, and a relevant research and practice agenda. The results of these Working Group meetings and discussions are presented in the position papers that follow.

\section{Cancer Center Cessation Initiative Coordinating Center and Expert Advisory Panel Members}

Betsy Rolland, PhD, MLIS, MPH ${ }^{1,2}$; Sarah D. Hohl, MPH, PhD ${ }^{1}$; Mara Minion $\mathrm{MA}^{1}$; Robert Adsit, MEd ${ }^{3}$; Margaret B. Nolan, MD, MS ${ }^{3,4}$; Danielle Pauk, $\mathrm{BS}^{1}$; Steven L. Bernstein, $\mathrm{MD}^{5}$; Jessica L. Burris, $\mathrm{PhD}^{6}$; Paul M. Cinciripini, $\mathrm{PhD}^{7}$ Anne Joseph, MD, $\mathrm{MPH}^{8}$; Jamie Ostroff, $\mathrm{PhD}^{9}$; Graham W. Warren, MD $\mathrm{PhD}^{10}$; and Michael C. Fiore, MD, MPH, MBA ${ }^{1,3}$

${ }^{1}$ Carbone Cancer Center, School of Medicine and Public Health; ${ }^{2}$ Institute for Clinical and Translational Research; ${ }^{3}$ Department of Medicine, Center for Tobacco Research and Intervention, School of Medicine and Public Health; and ${ }^{4}$ Department of Population Health Sciences, School of Medicine and Public Health, University of Wisconsin-Madison, Madison, Wisconsin; ${ }^{5}$ Department of Emergency Medicine, Geisel School of Medicine, Dartmouth College, Hanover, New Hampshire; ${ }^{6}$ Department of Psychology and Markey Cancer Center, University of Kentucky, Lexington, Kentucky; ${ }^{7}$ Department of Behavioral Science, The University of Texas MD Anderson Cancer Center, Houston, Texas; ${ }^{8}$ Department of Medicine, University of Minnesota, Minneapolis, Minnesota; ${ }^{9}$ Department of Psychiatry and Behavioral Sciences, Memorial Sloan Kettering Cancer Center, New York, New York; and ${ }^{10}$ Department of Radiation Oncology, Medical University of South Carolina, Charleston, South Carolina.

Submitted July 26, 2021; final revision received September 14, 2021 accepted for publication September 16, 2021.

Disclosures: P.M. Cinciripini has disclosed receiving nonmonetary research support from Pfizer. G.W. Warren has disclosed participating in research for the Agency for Healthcare Research and Quality and serving as a consultant for the Canadian Partnership Against Cancer. The remaining individuals have disclosed no relevant financial relationships.

Funding: This supplement was funded by the $\mathrm{C} 3 \mathrm{I}$ Coordinating Center contract from the National Cancer Institute (CRDF Award \#66590). In addition, authors received funding for their $\mathrm{C} 3$ l participation via a supplement to their NCl P30 cancer center support grant during the period 2017-2021.

Correspondence: Betsy Rolland, PhD, MLIS, MPH, University of WisconsinMadison, 610 Walnut Street, 370E, Madison, WI 53726.

Email: brolland@wisc.edu

\section{References}

1. American Cancer Society. Cancer Facts \& Figures 2020. Atlanta, GA: American Cancer Society; 2020.

2. National Center for Chronic Disease Prevention and Health Promotion (US) Office on Smoking and Health. The Health Consequences of Smoking-50 Years of Progress: a report of the Surgeon General. Atlanta, GA: Centers for Disease Control and Prevention (US); 2014.

3. Siegel RL, Miller KD, Fuchs HE, et al. Cancer statistics, 2021. CA Cancer J Clin 2021;71:7-33.

4. NCl-Designated Cancer Centers. NIH National Cancer Institute website. Accessed November 14, 2020. Available at: https://www.cancer.gov/ research/infrastructure/cancer-centers

5. Talluri R, Fokom Domgue J, Gritz ER, et al. Assessment of trends in cigarette smoking cessation after cancer diagnosis among US adults, 2000 to 2017. JAMA Netw Open 2020;3:e2012164-2012164.

6. Goldstein AO, Ripley-Moffitt CE, Pathman DE, et al. Tobacco use treatment at the U.S. National Cancer Institute's designated Cancer Centers. Nicotine Tob Res 2013;15:52-58.

7. Ramaswamy AT, Toll BA, Chagpar AB, et al. Smoking, cessation, and cessation counseling in patients with cancer: a population-based analysis. Cancer 2016;122:1247-1253.

8. Shields PG. New NCCN Guidelines: smoking cessation for patients with cancer. J Natl Compr Canc Netw 2015;13(Suppl 5):643-645.
9. Fiore $M C, D^{\prime}$ Angelo $H$, Baker $T$. Effective cessation treatment for patients with cancer who smoke-the fourth pillar of cancer care. JAMA Netw Open 2019;2:e1912264-1912264.

10. Henley SJ, Thomas CC, Sharapova SR, et al. Vital signs: disparities in tobacco-related cancer incidence and mortality - United States, 2004 2013. MMWR Morb Mortal Wkly Rep 2016;65:1212-1218.

11. Soulakova JN, Crockett LJ. Unassisted quitting and smoking cessation methods used in the United States: analyses of 2010-2011 tobacco use supplement to the current population survey data. Nicotine Tob Res 2017;20:30-39.

12. Croyle RT, Morgan GD, Fiore MC. Addressing a core gap in cancer care - the $\mathrm{NCl}$ Moonshot Program to help oncology patients stop smoking. N Engl J Med 2019;380:512-515.

13. D'Angelo H, Ramsey AT, Rolland B, et al. Pragmatic application of the RE-AIM framework to evaluate the implementation of tobacco cessation programs within $\mathrm{NCl}$-Designated Cancer Centers. Front Public Health 2020;8:221.

14. D'Angelo H, Rolland B, Adsit R, et al. Tobacco treatment program implementation at $\mathrm{NCl}$ Cancer Centers: progress of the $\mathrm{NCl}$ Cancer Moonshot-Funded Cancer Center Cessation Initiative. Cancer Prev Res (Phila) 2019;12:735-740.

15. D'Angelo $\mathrm{H}$, Webb Hooper $\mathrm{M}$, Burris $\mathrm{JL}$, et al. Achieving equity in the reach of smoking cessation services within the $\mathrm{NCl}$ Cancer Moonshot-Funded Cancer Center Cessation Initiative. Health Equity 2021;5:424-430. 